\title{
Modelagem probabilística de eventos de precipitação extrema no estado do Rio Grande do Sul
}

\author{
Tamara L. Caldeira ${ }^{1}$, Samuel Beskow ${ }^{2}$, Carlos R. de Mello ${ }^{3}$, \\ Lessandro C. Faria ${ }^{4}$, Mayara R. de Souza ${ }^{5} \&$ Hugo A. S. Guedes ${ }^{6}$
}

\section{Palavras-chave:}

chuvas intensas

distribuição de probabilidade

regionalização hidrológica

\begin{abstract}
R E S U M O
A gestão de cheias em bacias hidrográficas brasileiras deve ser discutida e priorizada, porém o cenário atual indica lacunas quanto às informações hidrológicas com variabilidade espacial e temporal condizentes. A modelagem probabilística de eventos extremos de precipitação, buscando a extrapolação para uma frequência e duração, pode servir como excelente ferramenta de análise e tomada de decisões. O objetivo principal deste trabalho foi analisar o ajuste de diferentes modelos probabilísticos a séries de precipitação máxima diária anual no Rio Grande do Sul. Séries pluviométricas de 342 estações foram ajustadas às distribuições Log-Normal a 2 e 3 parâmetros e Gumbel e a adequação foi realizada pelos testes de Kolmogorov-Smirnov e Qui-Quadrado. Todas as distribuições de probabilidade consideradas foram adequadas; entretanto, a distribuição Log-Normal a 3 parâmetros apresentou os melhores ajustes segundo os resultados do teste Qui-Quadrado. Os parâmetros das distribuições de probabilidades apresentaram variabilidade ao longo do estado e forte relação com a localização, sugerindo que a regionalização de chuvas intensas pode ser empregada no Rio Grande do Sul como excelente ferramenta de gestão.
\end{abstract}

\section{Key words:}

high-intensity rainfall probability distribution hydrological regionalization

\section{Probabilistic modelling of extreme rainfall events in the Rio Grande do Sul state}

\footnotetext{
A B S T R A C T
}

Flood management in Brazilian watersheds must be discussed and prioritized, however, the current scenario indicates that there are gaps in hydrological information with respect to its spatial and temporal variability. The probabilistic modelling of extreme rainfall events, having as goal to extrapolate values for a given frequency and duration, can be used as an excellent tool for analysis and decision-making. The main objective of this study was to analyse the adjustment of different probabilistic models for series of annual maximum daily rainfall in Rio Grande do Sul. Series of 342 rain gauges were adjusted to 2-parameter Log-Normal, 3-parameter Log-Normal and Gumbel probability distributions and goodnessof-fit tests were based on the Kolmogorov-Smirnov and Chi-Square procedures. It was found that all the distributions presented adequate results, however, 3-parameter LogNormal distribution had the best performance in accordance with the Chi-Square test. The parameters of probability distribution presented variability over the state and a pronounced relationship with the location of rain gauges. This suggests that regionalization of highintensity rainfall can be employed in Rio Grande do Sul as an excellent management tool.

Protocolo 054-2014 - 23/02/2014 • Aprovado em 31/10/2014 • Publicado em 26/01/2015

${ }^{1}$ Mestranda, PPG Recursos Hídricos/UFPel. Pelotas, RS. E-mail: tamaraleitzkecaldeira@gmail.com

${ }^{2}$ CDTec/Eng. Hídrica/UFPel. Pelotas, RS. E-mail: samuel.beskow@ufpel.edu.br (Autor correspondente)

${ }^{3}$ DEG/UFLA. Lavras, MG. E-mail: crmello@deg.ufla.br

${ }^{4}$ CDTec/Eng. Hídrica/UFPel. Pelotas, RS. E-mail: lessandro.faria@ufpel.edu.br

${ }^{5}$ Graduanda, Eng. Hídrica/UFPel. Pelotas, RS. E-mail: mayara.robertasouza@gmail.com

${ }^{6}$ CENG/Eng. Civil/UFPel. Pelotas, RS. E-mail: hugo.guedes@ufpel.edu.br 


\section{INTRODUÇÃo}

Estudos relacionados a chuvas intensas ou extremas são de grande relevância na gestão de recursos hídricos, visto que são aplicados frequentemente para o conhecimento do comportamento hidrológico de bacias hidrográficas no tocante ao controle de cheias e inundações, tal como para a estimativa de vazões de projeto para o dimensionamento de estruturas hidráulicas, de forma geral.

A intensidade de um evento de precipitação, além de estar diretamente relacionada com a duração, é função também da frequência com que este ocorre, sendo comumente empregados modelos probabilísticos buscando estimativa de valores vinculados a determinados níveis de probabilidades (Mello \& Silva, 2013).

Para a modelagem de chuvas intensas são utilizados dados pluviográficos ou pluviométricos sendo, de acordo com Mello \& Silva (2013), os pluviográficos os mais indicados haja vista que permitem a determinação das intensidades de precipitação associadas a diferentes durações; todavia, e face à dificuldade de se encontrar dados pluviográficos no Brasil, o procedimento mais comum é o emprego de dados pluviométricos por meio de séries de chuvas máximas diárias anuais (Back, 2001; Santos et al., 2009; Souza et al., 2012; Aragão et al., 2013).

Inúmeros são os modelos de distribuição de probabilidades aplicados a variáveis aleatórias contínuas, como é o caso de chuvas máximas diárias anuais, sendo que Naghettini \& Pinto (2007) recomendam, para o ajuste de valores extremos máximos, as distribuições Log-Normal a 2 parâmetros (LN2P), Log-Normal a 3 parâmetros (LN-3P), Assintótica de Valores Extremos do Tipo I, também conhecida como Gumbel, Assintótica de Valores Extremos do Tipo II, ou Fréchet, Generalizada de Valores Extremos e Pearson Tipo III.

A distribuição de Gumbel tem sido bastante utilizada para modelagem probabilística de valores extremos (Back, 2001; Silva et al., 2002, 2003; Back et al., 2011; Sampaio, 2011; Souza et al., 2012; Mello \& Viola, 2013); no entanto, a escolha e a definição da distribuição que melhor se ajusta ao conjunto de dados em análise devem ser realizadas comparando-se as frequências teóricas às frequências observadas por meio de testes estatísticos não paramétricos (Mello \& Silva, 2013). Ressalta-se que os resultados desses testes dependem, de acordo com Back (2001), dos parâmetros da distribuição de probabilidades e da equação de posição de plotagem podendo, ainda, indicar mais de uma distribuição adequada.

O grau de ajuste dos modelos probabilísticos é influenciado pela metodologia de estimativa dos parâmetros da distribuição. Naghettini \& Pinto (2007) relatam que o método dos Momentos é o mais simples para tal fim mas produz estimadores de baixa qualidade quando comparado ao método da Máxima Verossimilhança, principalmente para distribuições de probabilidade com três ou mais parâmetros.

O método da Máxima Verossimilhança, considerado o mais eficiente, produz estimadores de menor variância porém envolve equações em geral não lineares e implícitas. Ainda assim, para amostras pequenas, comumente utilizadas em estudos hidrológicos, o método dos Momentos pode estimar os parâmetros da distribuição com qualidade igual ou até mesmo superior às demais metodologias (Naghettini \& Pinto, 2007).
Diante do exposto, os objetivos deste estudo foram: i) ajustar as distribuições de probabilidade LN-2P, LN-3P e Gumbel a 342 séries de precipitação máxima diária anual de postos pluviométricos do Rio Grande do Sul; ii) verificar a distribuição de probabilidade que melhor representa os dados de chuva máxima diária anual e iii) subsidiar o estudo de chuvas intensas por meio de curvas intensidade-duraçãofrequência, visando à gestão de cheias no estado do Rio Grande do Sul.

\section{Material e Métodos}

Os dados empregados neste estudo foram obtidos junto ao banco de dados hidrometeorológicos da Agência Nacional de Águas (ANA), disponibilizado pelo Hidroweb - Sistema de Informações Hidrológicas, e correspondem a séries históricas de precipitação total diária no estado do Rio Grande do Sul.

Neste estudo foram considerados 342 postos pluviométricos, distribuídos espacialmente conforme ilustrado na Figura 1, para os quais os anos civis sem falhas proporcionavam no mínimo 10 anos de registros. Outros estudos acerca de chuvas intensas no Brasil (Back, 2001; Santos et al., 2009; Souza et al., 2012; Aragão, et al., 2013) admitem séries históricas seguindo um limiar mínimo de 10 a 15 anos de observações.

Os dados observados em escala diária permitiram computar séries de precipitação máxima diária anual, as quais foram ajustadas às distribuições de probabilidade Log-Normal a 2 parâmetros (LN-2P), Log-Normal a 3 parâmetros (LN-3P) e Gumbel que, dentre outras, são recomendadas por Naghettini \& Pinto (2007) e já foram aplicadas por diversos autores na modelagem probabilística de eventos extremos de chuva (Trefry et al., 2000; Back, 2001; Silva et al., 2002; Sansigolo, 2008; Ben-Zvi, 2009; Santos et al., 2009; Back et al., 2011; Souza et al., 2012; Aragão et al., 2013; Mello \& Viola, 2013).

Momentos de $1^{\text {a }}$ e $2^{\text {a }}$ ordens, considerando as distribuições LN-2P e Gumbel, e de $1^{\mathrm{a}}$, $2^{\mathrm{a}}$ e $3^{\mathrm{a}}$ ordens, distribuição LN3P, foram estimados com base no Método dos Momentos,

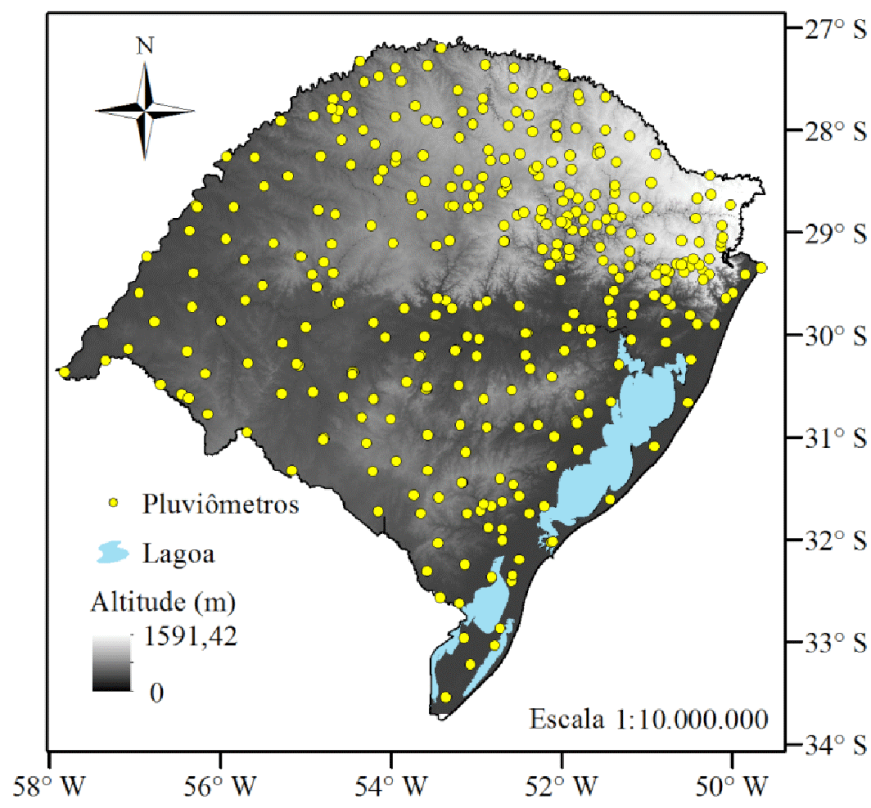

Figura 1. Distribuição espacial dos postos pluviométricos analisados neste estudo 
comumente empregado na estimativa de parâmetros em análises de variáveis hidrológicas (Back, 2001; Santos et al., 2009; Back et al., 2011; Souza et al., 2012; Aragão et al., 2013; Mello \& Viola, 2013).

A função densidade de probabilidade (FDP) da distribuição LN-2P, descrita em Naghettini \& Pinto (2007), é dada por:

$$
f_{X}(x)=\frac{1}{X \sigma_{\ln (X)} \sqrt{2 \pi}} e^{-\frac{1}{2}\left[\frac{\ln (X)-\mu_{\ln (X)}}{\sigma_{\ln (X)}}\right]}
$$

em que:

x - variável aleatória contínua que, nesta aplicação, corresponde à precipitação máxima diária anual

$\mu_{\ln (\mathrm{x})}$ - parâmetro relacionado ao $1^{\circ}$ momento amostral, estimado pela média dos dados logaritmizados

$\sigma_{\ln (\mathrm{x})}$ - parâmetro relacionado ao $2^{\circ}$ momento amostral, estimado pelo desvio padrão dos dados logaritmizados

Na distribuição LN-3P a FDP é semelhante àquela com 2 parâmetros, entretanto, da variável x se deduz a quantidade $\alpha$, que reflete o valor do limite inferior da amostra (Naghettini \& Pinto, 2007). Deste modo, a função densidade é dada por:

$$
f_{X}(x)=\frac{1}{(x-\alpha) \sigma_{Y} \sqrt{2 \pi}} e^{-\frac{1}{2}\left[\frac{\ln (x-\alpha)-\mu_{Y}}{\sigma_{Y}}\right]}
$$

em que:

$\mathrm{X} \quad$ - precipitação máxima diária anual

$\mathrm{Y} \quad$ - variável dada por $\mathrm{Y}=\ln (\mathrm{X}-\mathrm{\alpha})$

$\mu_{\mathrm{Y}}$ - parâmetro relacionado ao $1^{\circ}$ momento amostral, estimado pela média dos dados logaritmizados

$\sigma_{\mathrm{Y}} \quad$ - parâmetro relacionado ao $2^{\circ}$ momento amostral, estimado pelo desvio padrão dos dados logaritmizados

a - parâmetro relacionado ao $3^{\circ}$ momento amostral, estimado com base no coeficiente de assimetria

A função densidade da distribuição Gumbel, apresentada por Mello \& Silva (2013), é expressa por:

$$
f(x)=\alpha e^{-\alpha(x-\mu)-e^{-\alpha(x-\mu)}}
$$

em que:

x - precipitação máxima diária anual

a - parâmetro de escala da distribuição

$\mu$ - parâmetro de locação da distribuição

Os parâmetros da distribuição de Gumbel estimados pelo Método dos Momentos são dados por:

$$
\begin{gathered}
\hat{\alpha}=\frac{1,2826}{S} \\
\hat{\mu}=\bar{X}-0,45
\end{gathered}
$$

em que:

S - desvio padrão da série histórica
$\mathrm{X}$ - média da série histórica

A adequação dos modelos probabilísticos às séries de precipitação máxima diária anual foi verificada pelos testes de aderência Kolmogorov-Smirnov (KS) e Qui-Quadrado $\left(\lambda^{2}\right)$ os quais têm sido empregados para verificação de ajustes de distribuições de probabilidades na área de hidrologia (Sansigolo, 2008; Back et al., 2011).

A importação e a manipulação dos dados para a elaboração das séries de precipitação máxima diária anual, bem como o ajuste de modelos probabilísticos e os testes de aderência, foram conduzidos com o auxílio de um aplicativo computacional denominado "System of Hydrological Data Acquisition and Analysis" (SYHDA) (Beskow et al., 2013a; Correa et al., 2013).

O SYHDA é um aplicativo computacional desenvolvido no Laboratório de Simulação Hidrológica e Processamento de Dados, do Centro de Desenvolvimento Tecnológico, Universidade Federal de Pelotas. Consiste em um software idealizado para fornecer suporte à aquisição e análise de séries históricas de precipitação e vazão de modo integrado ao modelo hidrológico Lavras Simulation of Hydrology (LASH) (Beskow et al., 2011; Beskow et al., 2013b; Viola et al., 2013), facilitando a montagem do banco de dados de entrada para a simulação e a exploração dos resultados de saída, como também de modo independente, auxiliando na tomada de decisão em recursos hídricos (Beskow et al., 2013a).

\section{Resultados e Discussão}

Avaliando os cenários delineados pela distribuição LN-2P e pela distribuição Gumbel, foi possível identificar que os parâmetros foram estimados para todas as séries de precipitação máxima diária anual. O mesmo foi verificado por Back (2001) no ajuste dessas distribuições de probabilidades, também pelo método dos Momentos, para chuvas diárias extremas no estado de Santa Catarina.

Com relação à distribuição LN-3P, não foi possível estimar os parâmetros de 26 postos pluviométricos. De acordo com Naghettini \& Pinto (2007), a variável Log-Normal é positiva e tem coeficiente de assimetria superior a zero, fazendo com que seja bastante aplicada frente à modelagem de chuvas máximas anuais. Neste estudo o fato da distribuição LN-3P não ter sido ajustada para 26 postos pluviométricos pode ser explicado pelos coeficientes de assimetria das respectivas séries históricas, os quais foram negativos não sendo possível, para essas séries, estimar o parâmetro $\mu_{\mathrm{Y}}$ da distribuição.

No tocante à verificação do ajuste dos modelos probabilísticos às séries de precipitação máxima diária anual, em função dos resultados obtidos com o teste KS, pôde-se constatar que, considerando um nível de significância de 5\%, 2 séries não foram adequadas à distribuição de Gumbel, 9 não foram adequadas à distribuição $\mathrm{LN}-2 \mathrm{P}$ e 42 não foram adequadas à LN-3P.

Esta inadequação poderia ser atribuída à seleção da equação de posição de plotagem porém, ao verificar que as equações de Blom recomendadas para estudos associados a distribuições Normal e Log-Normal, e de Cunnane, recomendada para qualquer distribuição (Mello \& Silva, 2013), produziam erros entre frequências superiores àqueles encontrados com 
a equação de Weibull; constatou-se, então, que a escolha da equação de posição de plotagem não foi preponderante. Todavia, o teste KS é qualitativo e permite apenas concluir sobre a adequabilidade da distribuição de probabilidades testada, não oferecendo embasamento suficiente para comparar o ajuste entre diferentes distribuições (Mello \& Silva, 2013). Ressalta-se, ainda, que neste teste a distribuição teórica deve ser completamente conhecida, ou seja, seus parâmetros não devem ser estimados a partir dos elementos da amostra. Quando se desconhece a distribuição teórica, simulações de Monte Carlo demonstram que podem ocorrer rejeições indevidas da hipótese testada (Naghettini \& Pinto, 2007).

Embora seja um teste não paramétrico pouco rigoroso, KS vem sendo bastante utilizado para verificar a aderência das séries de precipitações máximas às distribuições de probabilidades, como se pode verificar nos trabalhos de Back (2001), Beijo et al. (2003), Back et al. (2011) e Aragão et al. (2013).

$O$ teste de aderência $\lambda^{2}$ é quantitativo e permite determinar a distribuição que melhor se ajusta a uma série de dados (Mello \& Silva, 2013). De acordo com os resultados do teste $\lambda^{2}$, pôde-se verificar que a hipótese de nulidade, ao nível de significância de $5 \%$, foi verdadeira para 329 postos pluviométricos, 293 e 317 , para as distribuições LN-2P, LN-3P e Gumbel, respectivamente.

A Figura 2 ilustra a localização dos postos pluviométricos e as respectivas distribuições de probabilidades que melhor se ajustaram às séries históricas, considerando-se o teste de adequação $\lambda^{2}$ ao nível de significância de $5 \%$.

Considerando os resultados acima expostos, a tendência de melhor ajuste pela LN-2P não é confirmada quando se realiza uma análise acurada dos resultados do teste $\lambda^{2}$ (Figura 2) pois a distribuição LN-3P gerou os menores valores $\lambda^{2}$ para 164 séries de dados, seguida da distribuição LN-2P, que melhor se ajustou a 114 postos e da distribuição Gumbel, cujo $\lambda^{2}$ foi inferior aos demais em 64 postos.

Os resultados obtidos divergem do que se discute acerca de distribuições de probabilidades para séries de dados de valores

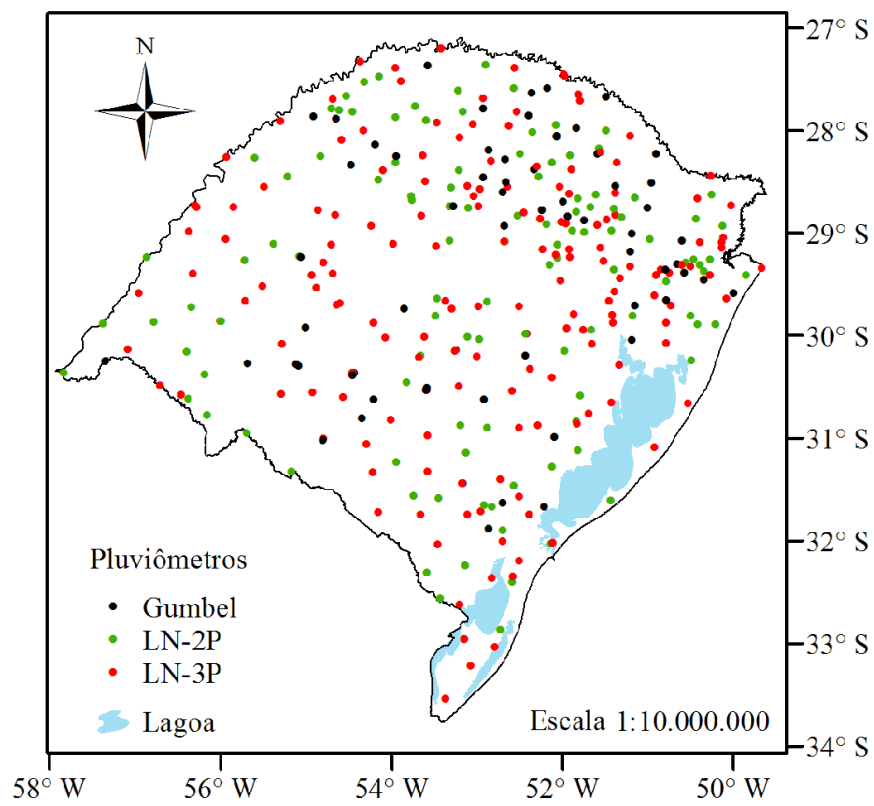

Figura 2. Distribuição de probabilidades mais adequada, ao nível de significância de $5 \%$, segundo o teste de aderência $\lambda^{2}$, para cada posto pluviométrico considerado neste estudo extremos máximos. Naghettini \& Pinto (2007) afirmam que a distribuição de Gumbel para máximos é a mais utilizada em estudos de relações intensidade-duração-frequência, chuvas intensas e vazões de enchente.

Back (2001) concluiu, ao testar diferentes distribuições de probabilidades para 100 postos pluviométricos do estado de Santa Catarina, que Gumbel foi o modelo que melhor se ajustou a $60 \%$ das séries de dados. Back et al. (2011) ajustaram a distribuição de probabilidades de Gumbel para séries de precipitação máxima diária anual a fim de estimar as equações de chuvas intensas para 13 estações pluviográficas de Santa Catarina. Mello \& Viola (2013) aplicaram a distribuição de Gumbel para o mapeamento de chuvas intensas no estado de Minas Gerais. Sampaio (2011) utilizou a distribuição de probabilidades de Gumbel para a espacialização dos coeficientes de chuvas intensas de 130 postos distribuídos em bacias hidrográficas do Rio Grande do Sul. Silva et al. (2002) comprovaram, para estimativa das equações de chuvas intensas para 19 postos pluviográficos do estado da Bahia, que o modelo de Gumbel foi o que apresentou melhor ajuste. Silva et al. (2003) testaram as distribuições de Gumbel, Log-Normal a 2 e 3 parâmetros, Pearson e Log-Pearson Tipo III para dados de pluviográficos de 10 estações do Tocantins, constatando que Gumbel foi a melhor ajustada. Souza et al. (2012) utilizaram a distribuição de probabilidade de Gumbel para obter equações de chuvas intensas para 74 postos pluviométricos do estado do Pará.

Com base no exposto ressalta-se que, de acordo com Back (2001) que muitos autores assumem a hipótese de que os dados analisados seguem modelo probabilístico de Gumbel sem testá-lo ou verificar se outra distribuição gera melhores ajustes.

Aragão et al. (2013) aplicaram as distribuições de Gumbel e Weibull aos dados de precipitação máxima anual de 48 postos de Sergipe e concluíram que Weibull proporcionou os melhores resultados. Ben-Zvi (2009) testou as distribuições de Gumbel, Log-Normal e Generalizada de Valores Extremos na determinação de curvas intensidade-duração-frequência para postos pluviométricos de Israel, constatando que o melhor ajuste ocorreu para o último modelo. Rahman et al. (2013) encontraram bons ajustes com as distribuições Generalizadas de Valores Extremos, Logística Generalizada e Generalizada de Pareto, ao modelar, por regiões hidrologicamente homogêneas, chuva máxima diária anual de 68 postos pluviométricos de Bangladesh. Trefry et al. (2000) testaram as distribuições Generalizada Logística, Generalizada de Valores Extremos, LN-3P, Pearson Tipo III e Generalizada de Pareto na regionalização de chuvas intensas para o estado de Michigan, Estados Unidos, concluindo que a Generalizada de Valores Extremos e a LN-3P geraram bons ajustes. Durrans \& Kirby (2004) aplicaram a distribuição Generalizada de Valores Extremos na regionalização de chuvas intensas no Alabama. Em seu estudo, Back (2001) verificou que as séries que apresentaram baixa assimetria e curtose se ajustaram melhor à distribuição de probabilidades LN-3P, enquanto que as séries com alta assimetria e curtose se ajustaram satisfatoriamente à distribuição Log-Pearson, seguida da LN-2P.

Observando os resultados obtidos por meio de uma análise estatística sobre os parâmetros estimados para cada posto pluviométrico (Tabela 1) pôde-se verificar, 
Tabela 1. Estatísticas descritivas básicas referentes aos parâmetros das distribuições de probabilidades considerando-se as séries adequadas, segundo o teste $\lambda^{2}$, ao nível de 0,05 de probabilidade

\begin{tabular}{|c|c|c|c|c|c|c|c|}
\hline Distribuição & $\begin{array}{c}\text { Número } \\
\text { de postos }\end{array}$ & Parâmetro & Mínimo & Máximo & Média & Desvio padrão & $\begin{array}{l}\text { Coeficiente de } \\
\text { variação (\%) }\end{array}$ \\
\hline \multirow{2}{*}{ LN-2P } & \multirow{2}{*}{329} & $\mu_{\ln (x)}$ & 4,00 & 4,84 & 4,49 & 0,13 & 2,98 \\
\hline & & $\sigma_{\ln (x)}$ & 0,13 & 0,84 & 0,27 & 0,07 & 24,39 \\
\hline \multirow{3}{*}{ LN-3P } & \multirow{3}{*}{293} & $\mu_{\gamma}$ & 2,75 & 8,11 & 4,52 & 0,86 & 18,96 \\
\hline & & $\sigma_{Y}$ & 0,01 & 1,05 & 0,33 & 0,22 & 66,36 \\
\hline & & $\alpha$ & $-3226,59$ & 76,45 & $-56,71$ & 258,49 & $-455,83$ \\
\hline \multirow{2}{*}{ Gumbel } & \multirow{2}{*}{317} & $\mu$ & 47,91 & 109,73 & 80,87 & 10,55 & 13,04 \\
\hline & & $\alpha$ & 0,02 & 0,13 & 0,05 & 0,02 & 29,84 \\
\hline
\end{tabular}

* Coeficiente de variação negativo em decorrência da média resultar em valor negativo

pelo coeficiente de variação, que o modelo probabilístico LN-3P foi o que apresentou a maior variabilidade entre os parâmetros, fato que pode ser atribuído ao ajuste da referida distribuição, que obteve o menor valor da estatística $\lambda^{2}$ para um número maior de séries, indicando que seus parâmetros

A.

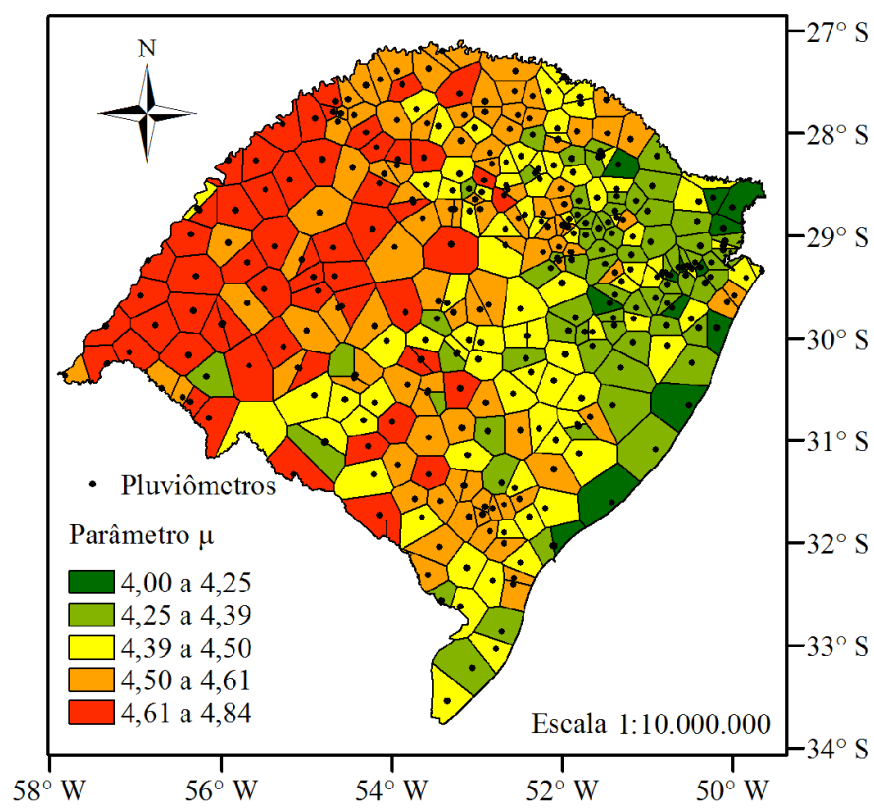

B.

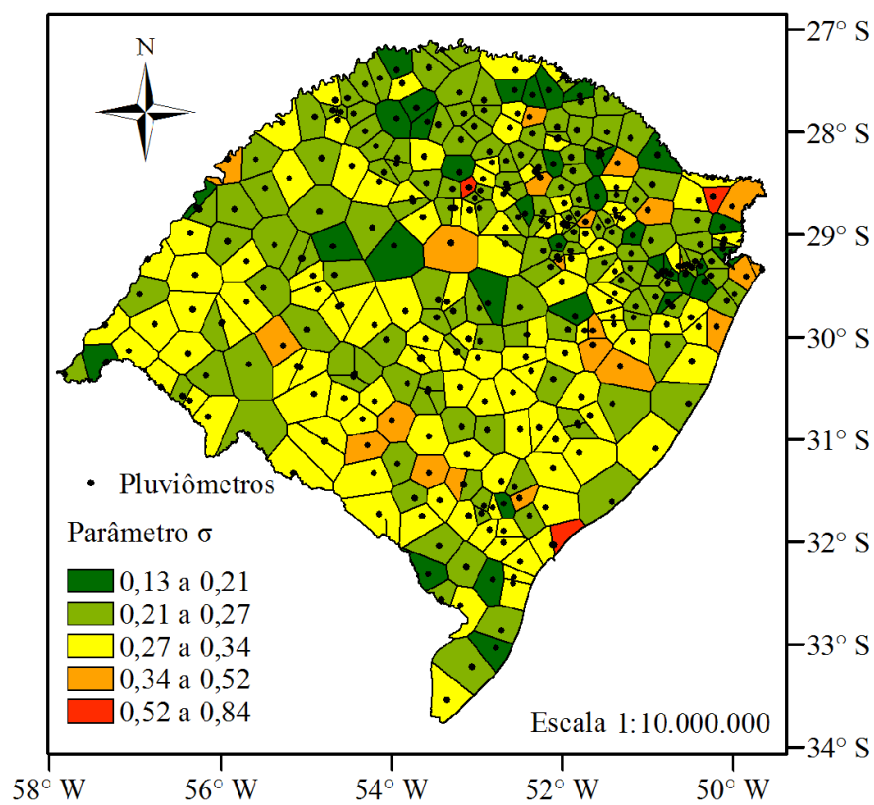

Figura 3. Distribuição espacial dos parâmetros $\mu$ (A) e $\sigma$ (B) da distribuição de probabilidades LN-2P representaram, de forma mais adequada, a distribuição de frequência das amostras.

Nas Figuras 3, 4 e 5 é apresentada a distribuição espacial dos parâmetros dos modelos probabilísticos LN-2P, Gumbel e LN-3P, respectivamente, apenas para os postos nos quais a

A.

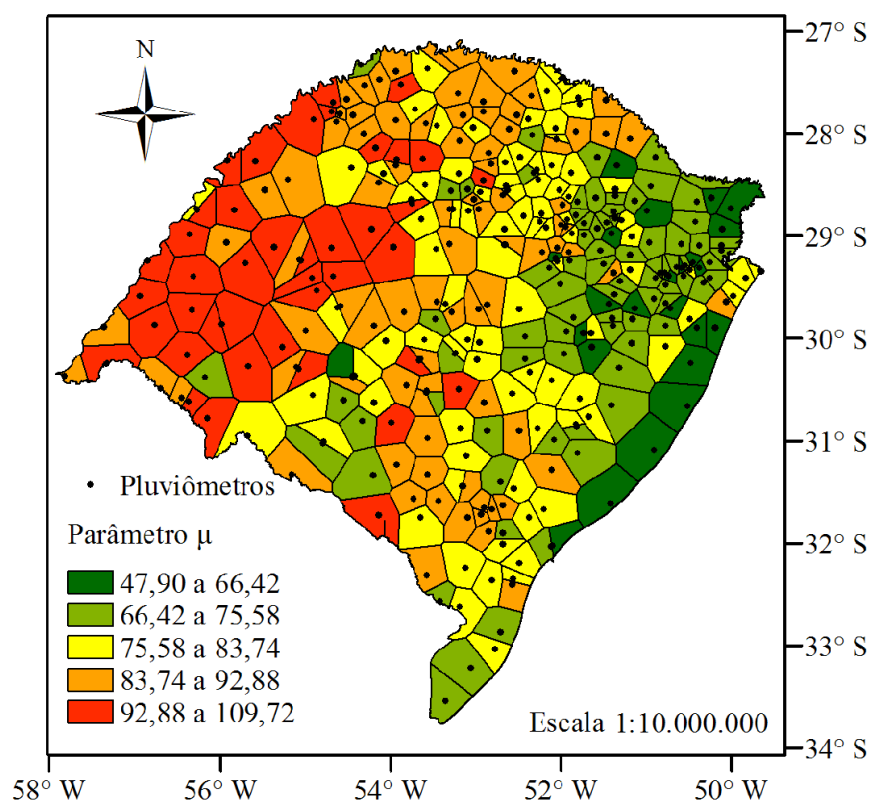

B.

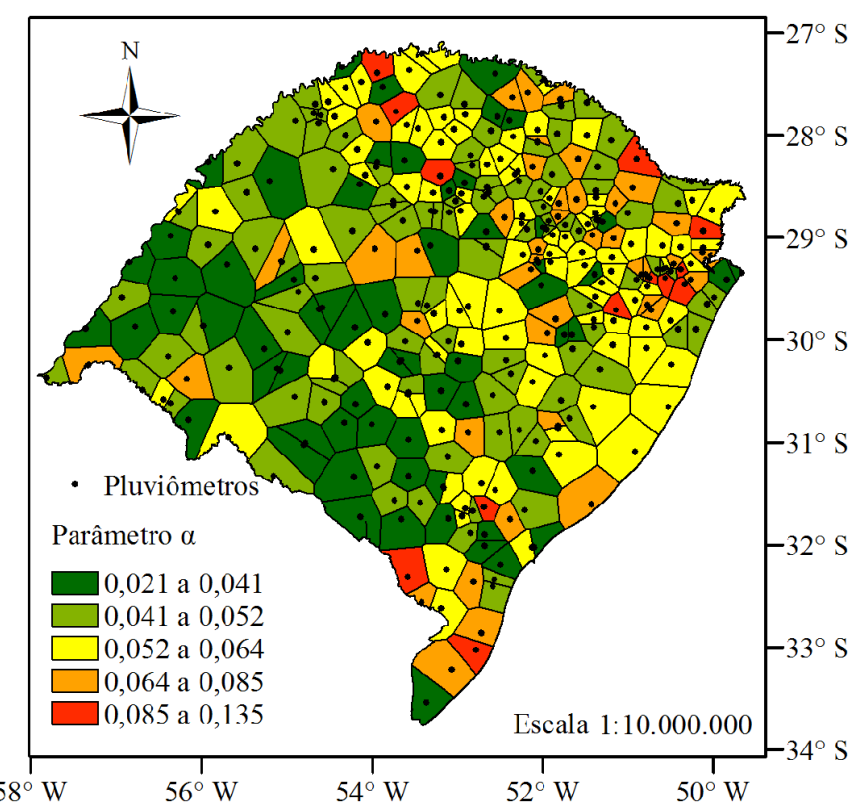

Figura 4. Distribuição espacial dos parâmetros $\mu$ (A) e $\alpha$ (B) da distribuição de probabilidades Gumbel 
A.

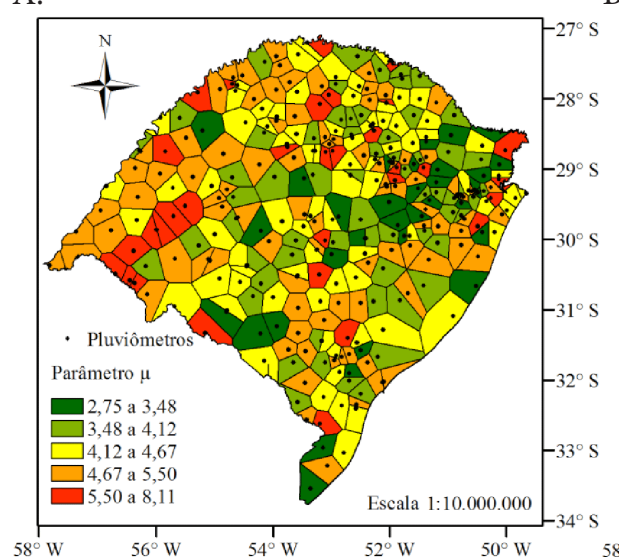

B.

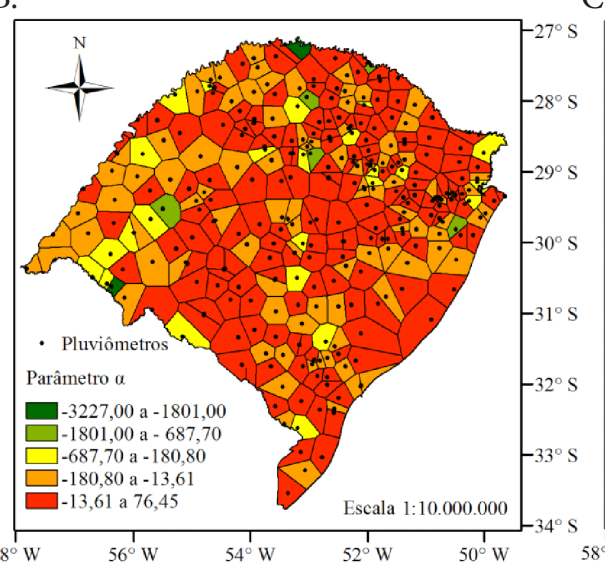

C.

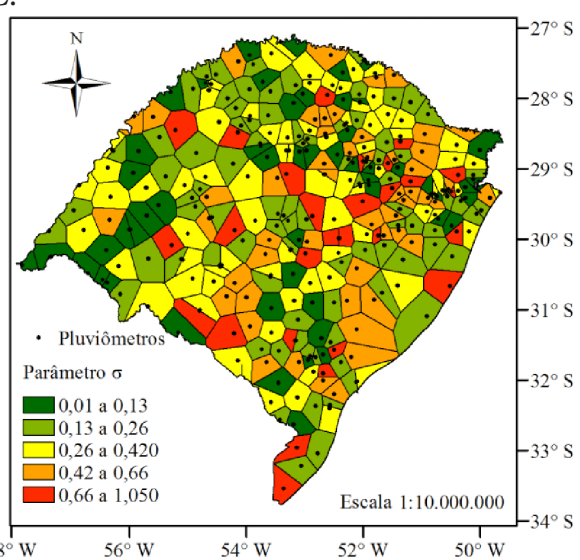

Figura 5. Distribuição espacial dos parâmetros $\mu(A)$, a (B) e $\sigma(C)$ da distribuição de probabilidades LN-3P

hipótese de nulidade foi aceita para o nível de significância de 0,05 , usando-se a metodologia dos polígonos de Thiessen para delimitar a área de influência de cada posto pluviométrico.

Analisando as Figuras 3, 4 e 5, verifica-se que os parâmetros das distribuições de probabilidades guardam relação direta com a localização espacial dos postos pluviométricos; entretanto, fica evidente que os parâmetros da distribuição LN-3P apresentaram maior variabilidade e dispersão espacial corroborando com os resultados apontados na Tabela 1 .

Os resultados obtidos dão indícios de que esforços científicos podem ser demandados com vistas à regionalização hidrológica destes parâmetros no intuito de disponibilizar uma excelente ferramenta de gestão no tocante às cheias no estado do Rio Grande do Sul haja vista que projetistas poderão estimar parâmetros para diferentes distribuições de probabilidade em determinado local de interesse, mesmo sem contar com séries de dados pluviométricos e/ou pluviográficos.

\section{Conclusões}

1. As distribuições de probabilidade avaliadas se mostraram adequadas para modelagem probabilística de eventos extremos de precipitação no estado do Rio Grande do Sul.

2. A distribuição LN-3P foi a que melhor se ajustou, de modo geral, às séries de precipitação máxima diária anual quando comparada aos modelos LN-2P e Gumbel.

\section{Agradecimentos}

Os autores agradecem à FAPERGS pelo apoio financeiro ao projeto de pesquisa 2082-2551/13 (Edital No 01/2013 Programa Pesquisador Gaúcho), pela bolsa de mestrado do primeiro autor e pela bolsa de iniciação científica do quinto autor, e ao CNPq, pelo apoio financeiro ao projeto de pesquisa 485279/2013-4 (Edital Universal MCTI/CNPq No 14/2013) e pela bolsa de produtividade do terceiro autor.

\section{Literatura Citada}

Aragão, R. de; Santana, G. R. de; Costa, C. E. F. F. da; Cruz, M. A. S.; Figueiredo, E. E. de; Srinivasan, V. S. Chuvas intensas para o estado de Sergipe com base em dados desagregados de chuva diária. Revista Brasileira de Engenharia Agrícola e Ambiental, v.17, p.243252, 2013. http://dx.doi.org/10.1590/S1415-43662013000300001
Back, A. J. Seleção de distribuição de probabilidade para chuvas diárias extremas do estado de Santa Catarina. Revista Brasileira de Meteorologia, v.16, p.211-222, 2001.

Back, A. J.; Henn, A.; Oliveira, J. L. R. Heavy rainfall equations for Santa Catarina, Brazil. Revista Brasileira de Ciência do Solo, v.35, p.2127-2134, 2011. http://dx.doi.org/10.1590/S010006832011000600027

Beijo, L. A.; Muniz, J. A.; Volpe, C. A.; Pereira, G. T. Estudo da precipitação máxima em Jaboticabal (SP) pela distribuição de Gumbel utilizando dois métodos de estimação dos parâmetros. Revista Brasileira de Agrometeorologia, v.11, p.141-147, 2003.

Ben-Zvi, A. Rainfall intensity-duration-frequency relationships derived from large partial duration series. Journal of Hydrology, v.367, p.104-114, 2009. http://dx.doi.org/10.1016/j. jhydrol.2009.01.007

Beskow, S.; Correa, L. L.; Mahl, M.; Simões, M. C.; Caldeira, T. L.; Nunes, G. S.; Hund, E. L.; Faria, L. C.; Mello, C. R. de. Desenvolvimento de um sistema computacional de aquisição e análise de dados hidrológicos. In: Simpósio Brasileiro de Recursos Hídricos, 20, 2013, Bento Gonçalves. Anais... Porto Alegre: ABRH, 2013a. CD Rom.

Beskow, S.; Mello, C. R. de; Norton, L. D.; Silva, A. M. Performance of a distributed semi-conceptual hydrological model under tropical watershed conditions. Catena, v.86, p.160-171, 2011. http://dx.doi.org/10.1016/j.catena.2011.03.010

Beskow, S.; Norton, L. D.; Mello, C. R. Hydrological prediction in a tropical watershed dominated by oxisols using a distributed hydrological model. Water Resources Management, v.27, p.341-363, 2013b. http://dx.doi.org/10.1007/s11269-0120189-8

Correa, L. L.; Caldeira, T. L.; Souza, M. R.; Simões, M. C.; Rodrigues, A. N.; Beskow, S. Módulo de aquisição e análise de dados hidrológicos para o modelo hidrológico LASH. In: Congresso de Iniciação Científica da Universidade Federal de Pelotas, 22, 2013, Pelotas. Anais... Pelotas: UFPel, 2013. CDRom

Durrans, S. R.; Kirby, J. T. Regionalization of extreme precipitation estimates for the Alabama rainfall atlas. Journal of Hydrology, v.295, p.101-107, 2004. http://dx.doi.org/10.1016/j. jhydrol.2004.02.021

Mello, C. R. de; Silva, A. M. Hidrologia: Princípios e aplicações em sistemas agrícolas. Lavras: UFLA, 2013. 455p. 
Mello, C. R. de; Viola, M. R. Mapeamento de chuvas intensas no estado de Minas Gerais. Revista Brasileira de Ciência do Solo, v.37, p.37-44, 2013. http://dx.doi.org/10.1590/S010006832013000100004

Naghettini, M.; Pinto, E. J. A. Hidrologia estatística. Belo Horizonte: CPRM, 2007. 552p.

Rahman, M. M.; Sarkar, S.; Najafi, M. R.; Rai, R. K. Regional extreme rainfall mapping for Bangladesh using L-Moment technique. Journal of Hydrologic Engineering, v.18, p.603-615, 2013. http:// dx.doi.org/10.1061/(ASCE)HE.1943-5584.0000663

Sampaio, M. V. Espacialização dos coeficientes das equações de chuvas intensas em bacias hidrográficas do Rio Grande do Sul. Santa Maria: UFSM, 2011. 115p. Tese Doutorado

Sansigolo, C. A. Distribuições de extremos de precipitação diária, temperatura máxima e mínima e velocidade do vento em Piracicaba, SP (1917-2006). Revista Brasileira de Meteorologia, v.23, p.341-346, 2008. http://dx.doi.org/10.1590/S010277862008000300009

Santos, G. G.; Figueiredo, C. C. de; Oliveira, L. F. C. de; Griebeler, N. P. Intensidade-duração-frequência de chuvas para o estado de Mato Grosso do Sul. Revista Brasileira de Engenharia Agrícola e Ambiental, v.13, p.899-905, 2009. http://dx.doi.org/10.1590/ S1415-43662009000700012
Silva, D. D. da; Gomes Filho, R. R.; Pruski, F. F.; Pereira, S. B.; Novaes, L. F. de. Chuvas intensas no estado da Bahia. Revista Brasileira de Engenharia Agrícola e Ambiental, v.6, p.362-367, 2002. http://dx.doi.org/10.1590/S1415-43662002000200030

Silva, D. D. da; Pereira, S. B.; Pruski, F. F.; Gomes Filho, R. R.; Lana, A. M. Q.; Baena, L. G. N. Equações de intensidade-duraçãofrequência da precipitação pluvial para o estado de Tocantis. Engenharia na Agriculta, v.11, p.7-14, 2003.

Souza, R. O. R. M.; Scaramussa, P. H. M.; Amaral, M. A. C. M. do; Neto, J. A. P.; Pantoja, A. V.; Sadeck, L. W. R. Equações de chuvas intensas para o estado do Pará. Revista Brasileira de Engenharia Agrícola e Ambiental, v.16, p.999-1005, 2012. http://dx.doi. org/10.1590/S1415-43662012000900011

Trefry, C. M.; Watkins, D. W.; Johnson, D. L. Development of Regional Rainfall Intensity-Duration-Frequency Estimates for the State of Michigan. In: Joint Conference on Water Resource Engineering and Water Resources Planning and Management, 2000, Minneapolis. Proceedings... Reston: ASCE, 2000. p.1-10.

Viola, M. R.; Mello, C. R. de; Beskow, S; Norton, L. D. Aplicability of the LASH Model for Hydrological Simulation of the GrandeRiver Basin, Brazil. Journal of Hydrologic Engineering, v.18, p.16931652, 2013. http://dx.doi.org/10.1061/(ASCE)HE.19435584.0000735 